\title{
Development of Productivity Evaluation Method to Improve Office Environment
}

\author{
Hiroshi Shimoda*1, Kyoko Ito*2, Yoko Hattori*1, Hirotake Ishii*1, Hidekazu \\ Yoshikawa*1, Fumiaki Obayashi*3, Masaaki Terano*3 \\ *1: Kyoto University, Gokasho, Uji, Kyoto, Japan, \\ *2:Osaka University, Toyonaka, Osaka, Japan, \\ *3:Matsushita Electric Work, Kadoma, Osaka, Japan. \\ shimoda@energy.kyoto-u.ac.jp
}

\begin{abstract}
The authors have developed a performance test, CPTOP (Cognitive Performance Test of Productivity), which combines 11 basic task testss to evaluate intelligent abilities of office workers in order to quantitatively and objectively evaluate their productivity by controlling office environment. However, in the previous experiment, it was found that the results of CPTOP contain in-fluences of learning effect and variation of difficulties depending on each prob-lem set. In this study, therefore, subjective experiments have been conducted in order to compensate these influences and a correction method has been proposed.
\end{abstract}

\section{Introduction}

Energy saving is one of the countermeasures of increasing green house gas emission caused by increasing recent worldwide energy consumption. Although the energy saving in factories and industrial plants has been promoted, that in office buildings has not improved enough. In Japan, the government has promoted that the temperature of air-conditioning system in the summer should be 28 degree Celsius and office workers are recommended to wear casual style cloths. However, the drop in productivity of office workers caused by the energy saving may extend their working time and this may consume more energy[1].

On the other hand, recent studies have indicated that improvement of office environment may improve the work productivity. However, the method which evaluates office productivity objectively and quantitatively has not been established yet. If such method is developed, it is expected that it can be utilized for the design of office room and the evaluation of energy consumption based on office work productivity.

From this viewpoint, the purpose of this study is to develop a performance test which is a simulated task set of office work in order to evaluate work efficiency of office workers objectively and quantitatively. 


\section{Productivity Evaluation Method}

\subsection{Conception of Office Productivity Evaluation}

Although there are various studies and proposals for evaluation of office productivity index[2-5], there is no objective and quantitative evaluation method which reflects actual office work and useful evaluation method has been required.

In order to develop an appropriate evaluation method of productivity, it is necessary to analyze actual office work and pick up the factors of the productivity index, and then to configure suitable evaluation method and indices based on the analysis.

Since the office work has lots of atypical variations, the abilities which are necessary to execute the office work are first revealed, then the office productivity is measured by evaluating the performance how much the abilities can be applied for the work. In ad-dition, in order to conform it to the actual office work, the configuration of the abilities which are required for each occupation category is revealed and the total office pro-ductivity is evaluated based on the result of performance and configuration.

In order to evaluate the improvement of office environment by using this method, the productivity index before improvement is compared with that after the improvement.

\subsection{Configuration of Productivity Indices}

In order to reveal the abilities necessary for the office work, the authors defined essential abilities based on the human abilities by Fleishman[6]. Among the abilities, 21 cognitive abilities such as "Oral Comprehension" and "Memorization" were picked up as the essential abilities for the office work. In addition, interviews and questionnaires were conducted to the office workers who were general affairs, engineers, managers and so on. Based on the results, 11 elementary abilities are picked up as shown in Table 1 and weight of each abilities were deduced for each occupation category[7]. The example of the result is shown in Figure 1. In order to calculate a office productivity of specified occupation category, the results of the performances of the abilities are given added weight to each performance based on the deduced weight.

Table 1. 11 elementary abilities necessary to perform office work

\begin{tabular}{lll}
\hline \multicolumn{2}{c}{ Abilities } \\
\hline 1.Oral Comprehension & 2.Written Comprehension & 3.Oral expression \\
4.Written expression & 5. Memorization & 6. Mathematical Reasoning \\
7. Deductive Reasoning & 8. Inductive Reasoning & 9. Information Ordering Category \\
10. Perceptual Speed & 11. Time Sharing & \\
\hline
\end{tabular}




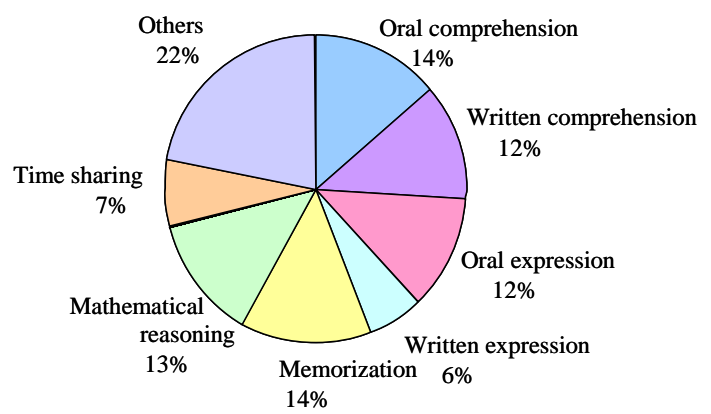

Fig. 1. An example of weights (clerical employee).

Based on this conception, the concrete productivity index can be calculated by equation (1).

This index can be calculated by the results of performance tests and weights of elementary abilities for a specific occupation and a specific office environment.

$$
\begin{aligned}
& \text { Office Productivity }=\sum_{i=1, j=1}^{I, J} W_{i, j} \cdot P_{i, j} \\
& W_{i, j}: \text { Weight s of elementary abilities } \\
& P_{i, j}: \text { Scores of performance test s for elementary abilities } \\
& i: \text { Occupation category } \\
& j: \text { Elementary abilities }
\end{aligned}
$$

\subsection{Performance Test System, CPTOP}

A performance test system, CPTOP (Cognitive Performance Tests for Office Productivity) has been developed to evaluate a productivity of office workers objectively and quantitatively based on the above productivity evaluation conception. CPTOP is a Web-based performance test system and it can be used by only PCs and Web browsers connected to the Internet as shown in Figure 2. 


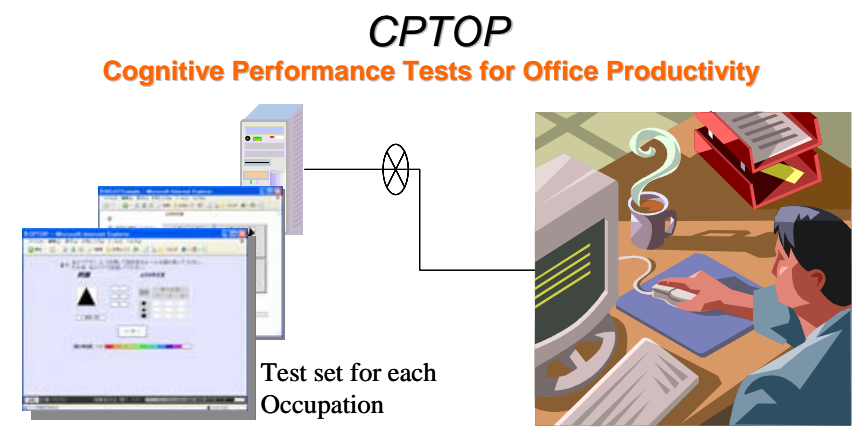

Fig. 2. Configuration of CPTOP

CPTOP consists of 11 sorts of task tests which correspond to the 11 elementary abilities. By changing the configuration of the test set and weights, the productivities in various office environments and situations can be evaluated.

Since the performance result of the task test are depending on each test such as throughput time and number of correct answers, the result score is standardized by dividing by the score average, then given the deduced weights to the standardized scores depending on the testee's occupation. Note that this test is to evaluate the productivity in a specific office environment, and NOT to evaluate the abilities of the individual workers

\subsection{Problem of CPTOP and Policy of its Improvement}

Since the purpose of CPTOP is to evaluate office environment by testing how much cognitive abilities can be performed in different office environments, the results of the same person in the same environment should be always the same. However, there might be a learning effect when repeating the same test, and difference of difficulties of the problems except automatically generated problems. If there are such leaning effect and difference of problem difficulties, it is difficult to evaluate cognitive abilities ex-actly.

The tests in which the learning effect appears are "Memorization", "Inductive Reasoning", "Information Ordering Category", "Perceptual Speed" and "Time Sharing”, which problems are automatically generated and difficulties of the problems are uniform. In this study, the authors assumed that the learning effect of each test is almost equal for anyone, and conducted a subject experiment to deduce the general learning curve for each test. And then, the learning effect will be removed by compensating each test result from the learning curve.

On the other hand, since the problems of "Oral Comprehension”, "Written Comprehension”, “Oral Expression”, "Written Expression”, “Mathematical Reasoning” and "Deductive Reasoning" cannot be generated automatically, they had been created in advance. Although a rule was laid down when creating the problem not to vary their difficulties, it is difficult to unify them in reality. In this study, therefore, the authors assumed that the difficulty of each problem is equal for anyone and conducted 
a sub-jective experiment to evaluate it. And then, the effect of difficulty variation will be removed by compensating each test result from the evaluated difficulties.

In the following chapters, two experiments to deduce the learning curve and to evaluate problem difficulties will be described.

\section{Experiment to Deduce Learning Curve}

\subsection{Objective of Experiment}

The objective of this experiment is to deduce the learning curves of five tests, "Memorization”, "Inductive Reasoning”, "Information Ordering Category”, "Perceptual Speed" and "Time Sharing", and propose the compensation method.

\subsection{Experimental Method}

The experiment was conducted in an experimental room of Advanced Research Laboratory of Kyoto University. The room temperature was adjusted to 25 degree Celsius and the intensity of illumination on the desk was 1000 lux.

The subjects of the experiment were 15 persons (6 males and 9 females, age: 2245, average age:30.9) who had experience to work with PC. They were explained the content and the purpose of the experiment and agreed to join it in advance.

In the experiment, the above five kinds of task tests of CPTOP were given to the subjects and the test results were measured. The experiment was conducted for three days for each subject. Since four sets of the task tests were given in each day, they performed 12 sets totally.

\subsection{Result of Experiment}

Figure 3 shows the average scores and their standard deviations of 12 sets in "Memorization”. The horizontal axis indicates number of the set from 1 to 12 , while the vertical axis indicates average scores and their standard deviations. From this graph, it was found that the scores are improved as the test set proceeded and the subjects learned the tests. Especially, the score improvement of "Memorization", "Deductive Reasoning" and "Time Sharing" tests were significant, and this indicated the necessity of com-pensation of learning effect.

Generally, it is assumed that the performance by the learning effect increases along with the experience and the performance will be saturated after infinite experience. Therefore, the following saturation model can be deduced. When supposing that the performance after $\mathrm{n}$ trials is $\mathrm{Pn}$, the initial performance is $\mathrm{P}$, the saturated performance is Plim and improvement ratio after a trial is $r$, the performance after $n$ trials can be described as equation (2). 


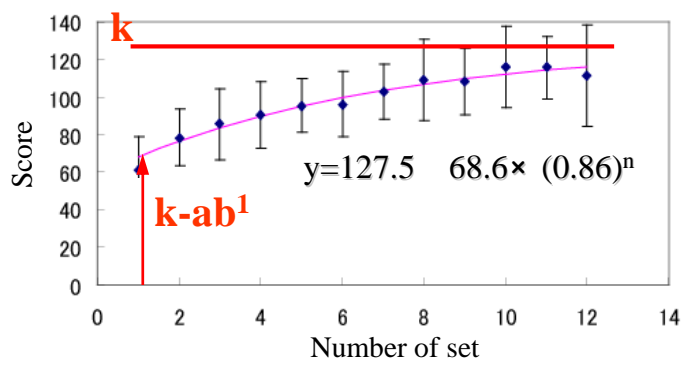

Fig. 3. Average scores and standard deviation of "Memorization"

$$
P_{n}=P_{\lim }-\left(P_{\lim }-P_{1}\right) \cdot(1-r)^{n-1}
$$

This equation can be simplified as learning curve as equation (3).

$$
y=k-a b^{x}
$$

Table 2 shows the coefficients which are calculated by minimum square method for the results of all the subjects.

Table 2. Coefficients of learning curve

\begin{tabular}{lccc}
\hline \multicolumn{1}{c}{ Task test } & $\mathrm{a}$ & $\mathrm{b}$ & $\mathrm{k}$ \\
\hline Memorization & 68.6 & 0.86 & 127.5 \\
Deductive Reasoning & 56.8 & 0.81 & 116.5 \\
Information Ordering Category & 35.5 & 0.91 & 118.6 \\
Cognitive Speed & 32.1 & 0.92 & 118.0 \\
Time Sharing & 66.1 & 0.86 & 128.6 \\
\hline
\end{tabular}

\subsection{Compensation Method of Learning Effect}

The conpensation method which removes the learning effect is proposed. In the equation (2), the saturated performance $\mathrm{k}$ is assumed as a standard score and the score is modified by the compensation ratio which is the saturated performance score divided by the performance of trial $n$. In other word, the conpensation ratio of trial $n$ can be described as follows;

$$
c_{n}=\frac{k}{k-a b^{n}}
$$


When supposing that the score of trial $n$ is Sn, the compensated task score, Tn will be described;

$$
T_{n}=c_{n} \times S_{n}
$$

\section{Difficulty Evaluation Experiment}

\subsection{Purpose of Experiment}

The purpose of this experiment is to evaluate the difficulties of problems for the task performance tests in which the problems can not be generated automatically, and propose the compensation method.

\subsection{Experimental Method}

The experiment was conducted at an experimental room of the advanced research laboratory in Kyoto University. The temperature of the room was controlled to 25 degree Celsius and the illumination on the desk was 1,000 lux.

In the experiment, five task tests excluding "Oral Expression" were given to the subjects. The experimental term was 6 days and about 20 problems of the five task tests were given to the subjects for one day, and totally 110 to 120 problems were given. Before the experiment, 5 problems of each test had been given to be accustomed to solve the problems. Between the task tests, they took enough rest to remove the fatigue effect.

Four subjects (2 males and 2 females, age:26-35, average age:29.3) joins the experiment who had experience to work with PC.

\subsection{Result of Experiment}

Figure 4 shows the average scores of each problem of "Written Comprehension". The horizontal axis indicates problem number, while the vertical axis indicates the average scores. In this graph, the high average score means easy problem, while the low average score means difficult problem. 


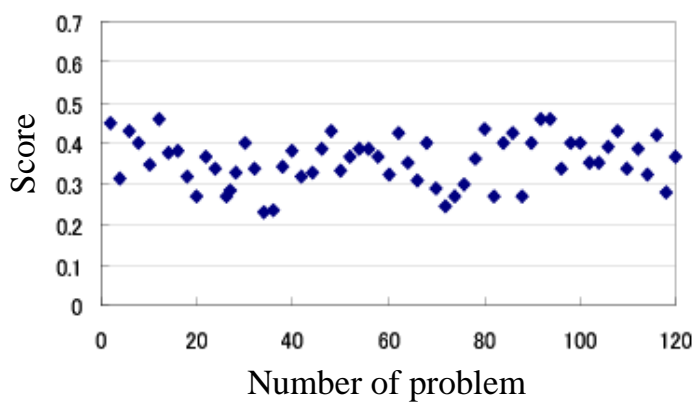

Fig. 4. Average score of each problem of "Written Comprehension".

\subsection{Compensation Method for Variation of Difficulties}

The difficulties of the problems can be described as the average score of all problems divided by the average score of each problem and the score of task test can be modified by the difficulty. In other word, the difficulty Di of problem i can be described using Tave which is the average score of each problem and Tall which is the average of all the problems as;

$$
D_{i}=\frac{T_{\text {all }}}{T_{\text {ave }}}
$$

When supposing that the score of the task test is $\mathrm{Si}$, the compensated score Ti can be described as;

$$
T_{i}=S_{i} \times D_{i}
$$

\section{Conclusion}

In this study, the authors have proposed an office productivity evaluation method CPTOP and the compensation methods of its learning effect and variation of difficulty of each problem by conducting two subject experiments for deducing the learning curve and evaluating the difficulties of each problem respectively. As the results of the first experiment, the learning curves were deduced and the compensation method of the test scores of "Memorization", "Deductive Reasoning", "Information Ordering Cate-gory", "Cognitive Speed" and "Time Sharing" was proposed by the learning curves. As the results of the second experiments, the difficulties of test problems were evaluated and the compensation method of test scores of "Oral Comprehension", "Written Comprehension", "Written Expression", 
“Mathematical Reasoning” and "Inductive Reasoning” was proposed by the evaluated difficulties.

In the future, the effectiveness of the proposed compensation methods will be confirmed by comparing CPTOP test score and the corresponding actual works in offices.

\section{References}

1. Lomonaco, G. and Miller,D (1997) Environmental Satisfaction, Personal Control and the Positive Correlation to Increased Productivity, Johnson Controls Inc..

2. Sensharma N. P. and Woods, J. E. (2000) An Extension of a Rational Model for Evaluation of Human Responses, Occupant Performance, and Productivity, Healthy Building 2000, Workshop 9.

3. Fisk W.J. et al. (2002) Worker Performance and Ventilation, Part 1,2, Proceedings of Indoor Air 2002, pp.784-795.

4. Kroner W.M. and Stark-Martin J.A. (1994) Environmentally Resposive Workstation and Office-Worker Productivity, ASHRAE Transactions, Vol.100, pp.750-755.

5. Wargocki P., et al. (2000) Productivity is Affected by the Air Quality in Offices, Healty Building 2000, pp.635-640.

6. Fleishman, E. A. and Reilly, M. E. (1992) Handbook of Human Abilities, Consulting Psychologists Press, pp.1-37.

7. Obayashi, F., et al. (2005) Objective Measurement Method for Productivity and Investigation on Environmental Factor, Proceedings of Human Computer Interaction International 2005, CD-ROM 